\title{
Comparison of time splitting and backsubstitution methods for integrating Vlasov's equation with magnetic fields
}

\author{
H. Schmitz and R. Grauer \\ Theoretische Physik I, Ruhr-Universität Bochum, 44780 Bochum, Germany
}

\begin{abstract}
The standard approach for integrating the multidimensional Vlasov equation using grid based, conservative schemes is based on a time splitting approach. Here, we show that although the truncation error is of second order, time splitting can introduce systematic heating of the plasma. We introduce a backsubstitution method, which not only avoids this deficiency but also is computationally less expensive. The general approach is demonstrated in conjunction with Boris' scheme for evaluating the characteristics.
\end{abstract}

Key words: Vlasov simulations, method of characteristics PACS: 02.70.-c, 52.25.Dg, 52.65.Ff, 52.25.Xz

\section{Introduction}

Vlasov's equation is fundamental for numerous problems in plasma theory. This kinetic equation describes the behaviour of the single particle distribution functions of a collisionless plasma under the influence of electric and magnetic fields. Coupled with the equations for the electromagnetic fields and the evaluation of the moments of the distribution functions one obtains a highly nonlinear system of differential and integral equations. Only a few very simple problems can be solved analytically. For this reason numerical simulations of Vlasov's equation have become an important tool for theoretical plasma physics.

One type of computer simulation approach integrates the distribution function directly on a high-dimensional numerical grid in phase space. Here one dimension is needed for every space component and for every velocity component. Following the original work by Cheng and Knorr [1] much progress has been made on improving the accuracy of the advection schemes. 
The integration of the distribution function can be carried out in a number of different ways: The simplest schemes are finite difference schemes. They are relatively easy to implement but suffer from numerical instabilities and nonpositivity of the distribution function. Conservation laws such as the conservation of particle number can be implemented but complicate the scheme greatly [2]. Conservative methods, on the other hand, discretise the distribution function by integrating over the numerical grid cells [3]. The advantage of these methods lies in the fact that the particle number is naturally conserved and no artificial sources or sinks of particles are introduced. Semi-Lagrangian methods (e.g. [4]) follow the characteristics backwards and interpolate the distribution function at the origin of the characteristic. The interpolated value is then transported forward to the grid points. Semi-Lagrangian methods do not naturally conserve the particle number but can easily be made to preserve positivity.

Most of the above methods are, however, developed for a one-dimensional advection problem. When used for a one-dimensional electrostatic system in which the physical phase space is two-dimensional, a time splitting method is employed which was already proposed in [1]. Although Semi-Lagrangian methods in principle allow to integrate the distribution function directly on the high-dimensional grid, the time splitting technique is also used to simplify the computation [5]. The general idea is that in higher dimensions this time splitting can be generalised in a straightforward way [6].

We will show in this paper that, when including a magnetic field, this simple time splitting - although second order - can cause dissipation due to errors which are always in the same direction. This implies that the temperature of the system will increase systematically. We will also present an alternative method which we named backsubstitution method. The backsubstitution method not only eliminates the problems of the time splitting method but is also computationally less expensive.

In section 2 we will present the basic underlying equations. Section 3 will describe the time splitting method and show how a systematic error develops. In section 4 we will present the backsubstitution method which we will apply to Boris' scheme in section 5. Section 6 discusses simulations of Bernstein waves using the different schemes to provide a comparison. Section 7 gives some concluding remarks.

\section{General Problem}

The basis of the kinetic plasma description is the distribution function $f(\mathbf{x}, \mathbf{v}, t)$ which expresses the particle density in phase space. Here 
$f(\mathbf{x}, \mathbf{v}, t) d^{3} x d^{3} v$ is the number of particles in a phase space volume $d^{3} x d^{3} v$ located at $(\mathbf{x}, \mathbf{v})$ at time $t$. In a collisionless plasma the evolution of the distribution function is given by Vlasov's equation

$$
\frac{\partial f}{\partial t}+\mathbf{v} \cdot \nabla_{\mathbf{x}} f+\frac{q}{m}(\mathbf{E}+\mathbf{v} \times \mathbf{B}) \cdot \nabla_{\mathbf{v}} f=0
$$

where $\mathbf{E}$ and $\mathbf{B}$ are the electric and magnetic fields which have to be determined self-consistently. Vlasov's equation describes the advection of values of the distribution function along particle characteristics given by Newton's law of motion.

One central property of Vlasov's equation is the conservation of the phase space density, which directly translates into a conservation of mass and charge in a closed system. For this reason it is natural to use a conservative scheme for simulating Vlasov's equation (for 1-dimensional schemes, see e.g. [7]). Today, a diversity of Eulerian schemes, all with high accuracy and different advantages and disadvantages, are available (see e.g. [5,8] and references therein). These schemes normally solve the one-dimensional advection problem,

$$
\partial_{t} f(x, t)+u \partial_{x} f(x, t)=0
$$

By integrating over a finite time step one obtains

$$
\int_{x_{i-1 / 2}}^{x_{i+1 / 2}} f\left(x, t^{n+1}\right) d x=\int_{X\left(t^{n}, t^{n+1}, x_{i-1 / 2}\right)}^{X\left(t^{n}, t^{n+1}, x_{i+1 / 2}\right)} f\left(x, t^{n}\right) d x .
$$

Here $X(s, t, \xi)$ denotes the characteristic with parameter $s$ that satisfies $X(t, t, \xi)=\xi$.

For the one dimensional, electrostatic Vlasov-Problem

$$
\frac{\partial f}{\partial t}+v \partial_{x} f+\frac{q}{m} E \partial_{v} f=0
$$

a splitting technique is then usually employed. Here one integrates the advection in the $x$-direction by $\Delta t / 2$, then in $v$-direction by $\Delta t$ and then again in $x$-direction by $\Delta t / 2$. This produces a second order scheme which can be written as

$$
T_{x}(\Delta t / 2) T_{v}(\Delta t) T_{x}(\Delta t / 2)
$$

Here $T_{k}$ denotes the numeric advection operator in the $k$-dimension. 


\section{Time splitting}

The success of the time splitting for the one dimensional electrostatic problem motivates a common suggestion to extend the splitting technique to treat higher-dimensional systems. Since the spatial dimensions are completely independent of each other this results in the following second order scheme for the full three dimensional system

$$
\begin{array}{r}
T_{x}(\Delta t / 2) T_{y}(\Delta t / 2) T_{z}(\Delta t / 2) \\
T_{v_{x}}(\Delta t / 4) T_{v_{y}}(\Delta t / 2) T_{v_{x}}(\Delta t / 4) T_{v_{z}}(\Delta t) T_{v_{x}}(\Delta t / 4) T_{v_{y}}(\Delta t / 2) T_{v_{x}}(\Delta t / 4) \\
T_{x}(\Delta t / 2) T_{y}(\Delta t / 2) T_{z}(\Delta t / 2) .
\end{array}
$$

In each of these sub-steps a one dimensional transport equation of type (2) is solved. For each of these equations the characteristics are calculated and then projected onto the corresponding direction. This implies that even for a hypothetical exact one-dimensional integration scheme the characteristics are still only approximated by a second order time splitting scheme. For the following discussion, we will consider only the velocity part of the integration scheme since this determines how well the particle temperatures are described.

For a purely electrostatic system the above integration behaves well and errors only occur due to repeated application of the advection scheme. The reason for this is the independence of the change of the velocity component $\Delta v_{k}$ on the velocity $v_{k}$. With a magnetic field, however, the change of velocity $\Delta v_{k}$ over a finite time step does depend on the velocity $v_{k}$. Here we assume that the integration scheme for the characteristics is at least second order. To investigate the error caused by this method, we take the exact characteristic in $\mathbf{v}$-space and approximate it using the time splitting scheme. During integration of the characteristic, the electromagnetic fields are assumed to be constant. Without loss of generality we let $\mathbf{B}=B \hat{\mathbf{z}}$ and move the origin in velocity space to $\mathbf{v}_{0}=\mathbf{E} \times \mathbf{B}$. For simplicity we assume $E_{z}=0 . E_{z} \neq 0$ would only add a constant acceleration in the $v_{z}$-direction, and leads to the same result.

In this setup the characteristics in velocity space are simple concentric circles around the origin and we can neglect the $v_{z}$ coordinate completely. During the time interval $\Delta t$ the whole $v_{x}, v_{y}$-plane rotates by an angle $\phi=\Delta t q B / m$. Taking a velocity

$$
\mathbf{v}=\left(v_{x}, v_{y}\right)
$$

we split the rotation into three steps according to the time-splitting scheme

$$
T_{v_{x}}(\Delta t / 2) T_{v_{y}}(\Delta t) T_{v_{x}}(\Delta t / 2) .
$$


This results in the following

$$
\begin{aligned}
\mathbf{v}^{a} & =\left(v_{x} \cos (\phi / 2)-v_{y} \sin (\phi / 2), v_{y}\right), \\
\mathbf{v}^{b} & =\left(v_{x}^{a}, v_{y}^{a} \cos (\phi)+v_{x}^{a} \sin (\phi)\right), \\
\mathbf{v}^{\text {new }} & =\left(v_{x}^{b} \cos (\phi / 2)-v_{y}^{b} \sin (\phi / 2), v_{y}^{b}\right) .
\end{aligned}
$$

Inserting $\mathbf{v}^{a}$ into $\mathbf{v}^{b}$ and then $\mathbf{v}^{b}$ into $\mathbf{v}^{\text {new }}$ results in a lengthy expression for $\mathbf{v}^{\text {new }}$. Taking the norm of $\mathbf{v}^{\text {new }}$ and expanding this expression for small angles $\phi$, i.e. small time steps $\Delta t$, gives

$$
\left(v^{\text {new }}\right)^{2}=v_{x}^{2}+v_{y}^{2}-\left(v_{x}^{2}+\frac{v_{y}^{2}}{2}\right) \phi^{2}+\mathcal{O}\left(\phi^{3}\right) .
$$

By construction this is, of course, second order in $\phi$. However, one can see that the second order error is always negative and thus introduces a systematic error.

The time splitting method (8) can also be interpreted as performing the individual steps (9)-(11) in first order in $\phi$. This corresponds to

$$
\begin{aligned}
\mathbf{v}^{a} & =\left(v_{x}-v_{y} \phi / 2, v_{y}\right), \\
\mathbf{v}^{b} & =\left(v_{x}^{a}, v_{y}^{a}+v_{x}^{a} \phi\right), \\
\mathbf{v}^{\text {new }} & =\left(v_{x}^{b}-v_{y}^{b} \phi / 2, v_{y}^{b}\right) .
\end{aligned}
$$

With respect to equation (2) this scheme is obtained by holding $u$ constant for each step. Taking the square of this $\mathbf{v}^{\text {new }}$ results in

$$
v^{\text {new } 2}=v_{x}^{2}+v_{y}^{2}+\frac{\phi^{3}}{2} v_{x} v_{y}+\mathcal{O}\left(\phi^{4}\right) .
$$

One can observe that in this case the second order disappears, and the third order error is not systematic, but depends on the signs of $v_{x}$ and $v_{y}$. In the following we will refer to eqs (9)-(11) as scheme $A$ and (13)-(15) as scheme $B$.

In Fig. 1 the error of the magnitude $|\mathbf{v}|$ of the velocity after a quarter gyration is plotted against the rotation angle $\Delta \varphi$ of the individual step. The error is normalised to the initial velocity. The solid line shows the result of scheme A while the dashed line shows the result of scheme B. The dotted line represents the result of scheme $\mathrm{B}$ with alternating order of the $v_{x}-v_{y}$ integration. For scheme $\mathrm{A}$, a total error of about $2.5 \%$ is accumulated after a quarter gyration when $\Delta \varphi \approx 0.045$. After a full gyration the error sums up to $10 \%$ (not shown). This value of $\Delta \varphi$ corresponds to roughly 140 integration steps for the full circle. Using less steps, i.e. larger $\Delta \varphi$ results in even larger errors.

To understand the direction of the error we note, that in eq. (3) the characteristics are integrated backwards from a grid point to obtain the source of 


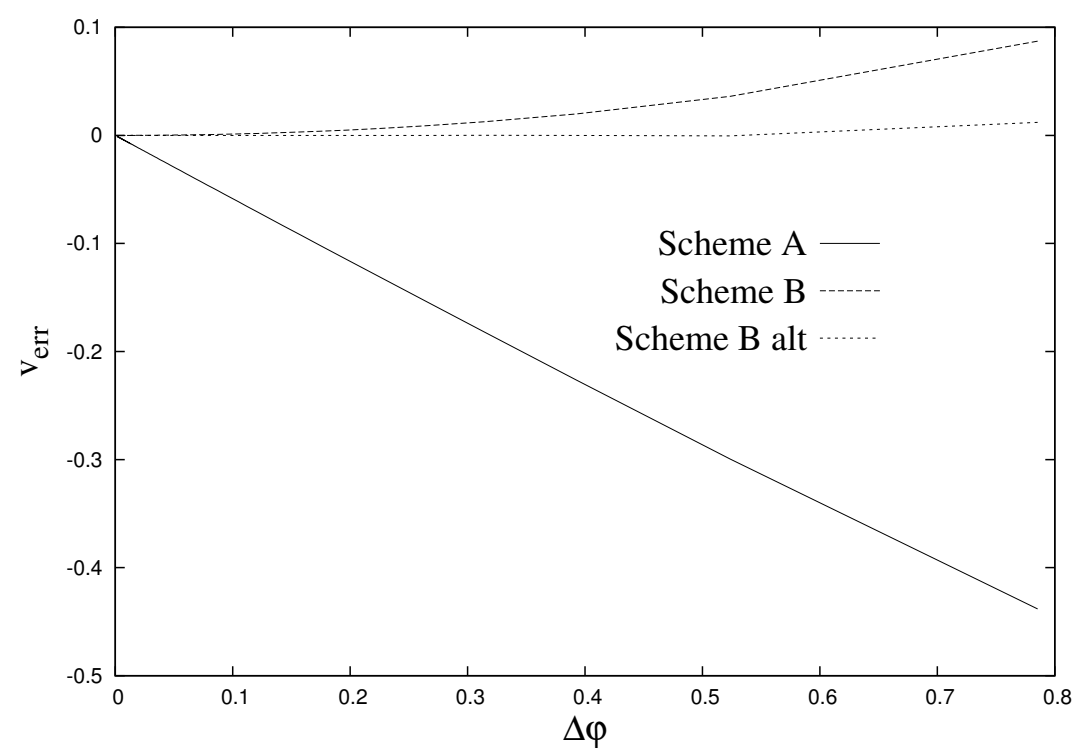

Fig. 1. Error $v_{e r r}$ of the magnitude of the velocity after a quarter gyration depending on the angle of an individual step. Curves are plotted for the schemes A, B and scheme $\mathrm{B}$ with alternating order of the $v_{x}-v_{y}$ integration.

the distribution function for that grid point. The distribution function is then transported from that source to the grid in some manner that depends on the numerical scheme. The negative sign in the second order of eq. (12) implies that the source is always located closer to the rotation centre than the grid point. Thus the values of the distribution function are transported outwards from the rotation centre. This results in an effective heating of the distribution function.

Using scheme $\mathrm{B}$ the errors are smaller but not zero. Here an error of $2 \%$ is observed when $\Delta \varphi \approx 0.4$. This is equivalent to roughly 16 steps for a full gyration. Because the direction of the error in scheme B depends on the values of $v_{x}$ and $v_{y}$, one can further increase the accuracy by alternating the order of the splitting. In the two dimensional case considered here this simply implies alternating the roles of $v_{x}$ and $v_{y}$. Using the alternating scheme the overall error in the velocity magnitude is reduced to almost zero. However, when looking at the relative phase error after a quarter gyration (Fig. 2) no significant improvement can be observed. While scheme A still shows the largest error, the errors for scheme $\mathrm{B}$ with and without alternating oder of integration are roughly comparable up to a $\Delta \varphi$ of 0.5 . For this value of $\Delta \varphi$ the phase error is approximately $1 \%$.

When the Vlasov equation is solved on a discretised grid errors are worse but the main sources of these errors are highlighted by the above analytical argument. 


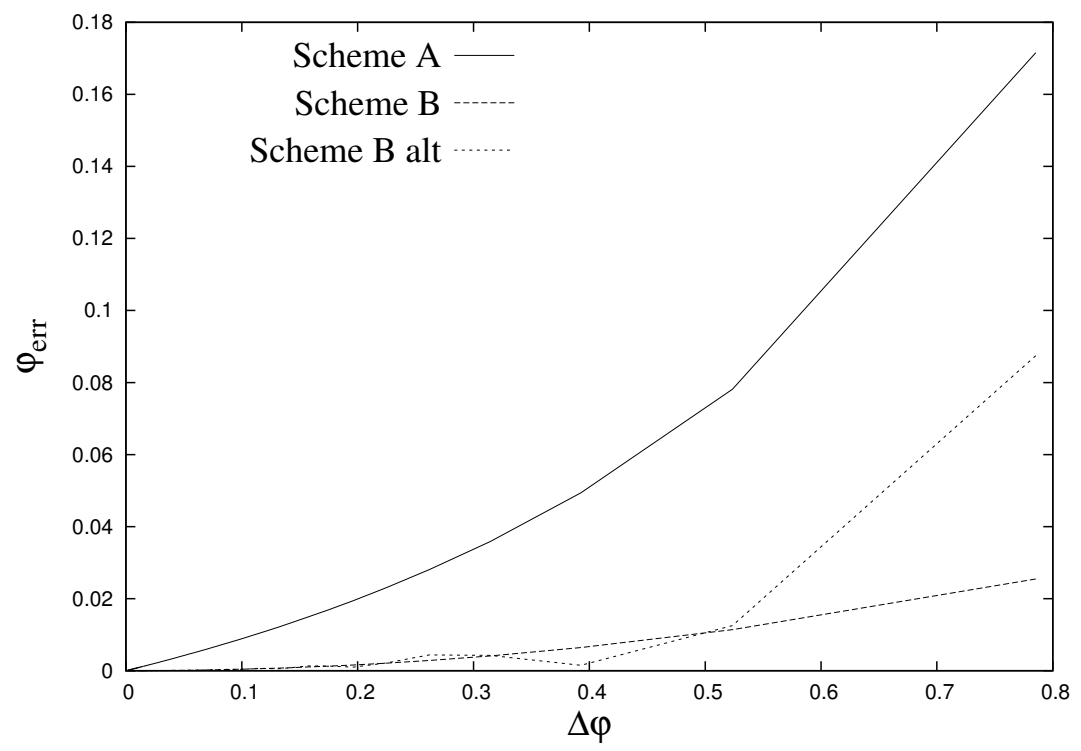

Fig. 2. Error $\phi_{\text {err }}$ of the phase of the velocity after a quarter gyration depending on the angle of an individual step. Curves are plotted for the schemes A, B and scheme B with alternating order of the $v_{x}-v_{y}$ integration.

\section{Backsubstitution}

In this section we want to present an alternative method for integrating Vlasov's equation that does not suffer from the above drawbacks. Here we will present first the general idea of this backsubstitution method and then write down the equations for the general system described above.

Suppose we are given a one dimensional integration scheme for the transport eq. (2). To create a scheme for the integration of the three-dimensional velocity space there is no other choice but to split the full three-dimensional problem into a number of one-dimensional substeps. For each of these substeps the characteristics will be calculated and then projected onto the direction of the advection step. We still have the freedom, which characteristics to integrate and in which order to integrate them.

To start with, let us again consider the standard case described in the last section. Our aim is to formulate a splitting scheme in which the characteristics are integrated exactly, and which uses the minimum number of integration steps. Since we can ignore the $v_{z}$-direction, this means we want only two integration steps, one for $v_{x}$, and one for $v_{y}$.

The distribution function is first shifted in the $v_{x}$, and then in the $v_{y}$ direction. Figure 3 illustrates the first step while Figure 4 illustrates the second step. Both shifts together should transport the value of the distribution function from a source point $S=\left(S_{x}, S_{y}\right)$ of a characteristic to its destination point 


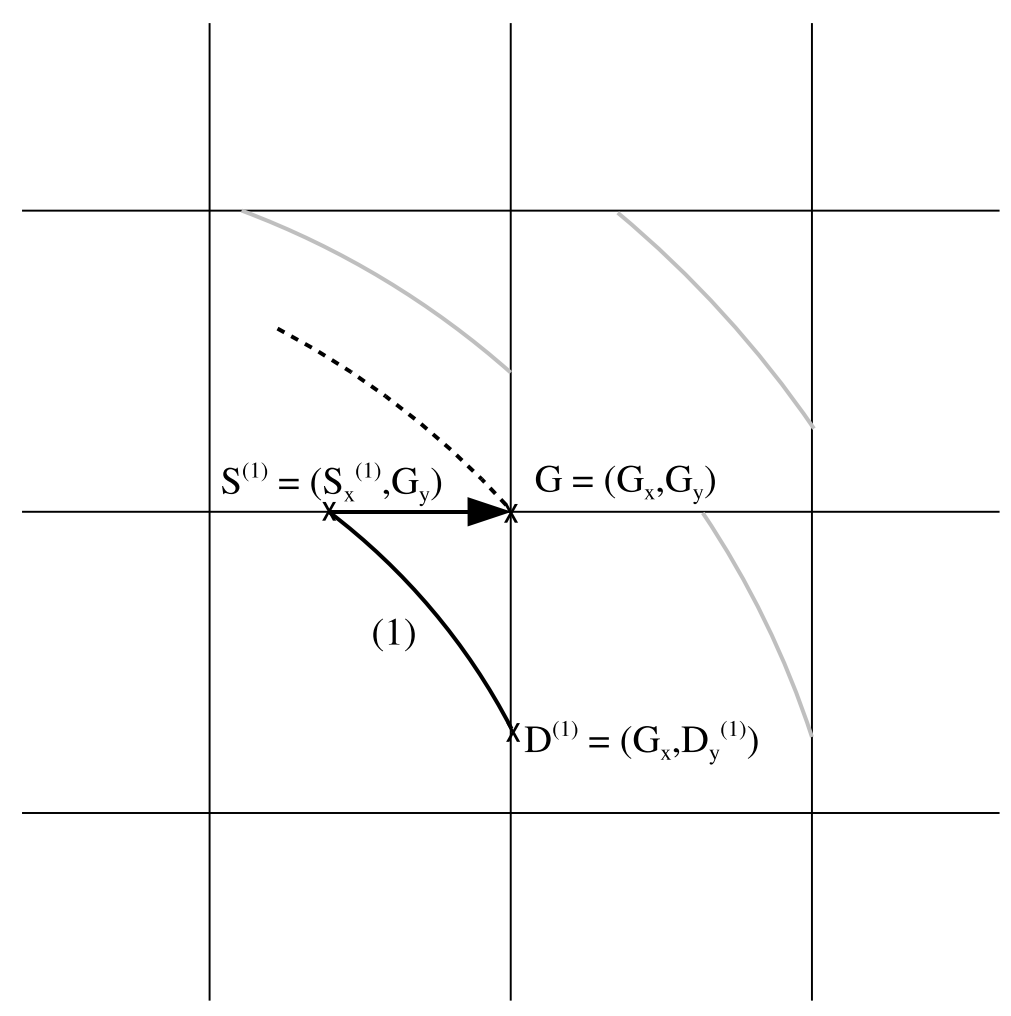

Fig. 3. Schematic diagram of the first step of integration of the characteristics using the backsubstitution algorithm. The distribution function is shifted in the $v_{x}$-direction from the source point $S^{(1)}$ of the characteristic to the grid point $G$. Gray lines show corresponding characteristics in the other cells. The dashed line is the characteristic ending on $G$ which is important in the second step.

$D=\left(D_{x}, D_{y}\right)$ with $S=X(t-\Delta t, t, D)$. Here the indices $x, y$, and $z$ are used to denote the velocity components $v_{x}, v_{y}$ and $v_{z}$. This means that we aim to find a scheme such that

$$
f^{\text {new }}\left(D_{x}, D_{y}\right)=f^{\text {old }}\left(S_{x}, S_{y}\right)
$$

In the first step the shift in $v_{x}$ has to transport $f$ from $S$ to an intermediate point $\left(D_{x}, S_{y}\right)$. In the semi-Lagrangian schemes which we are considering here, the characteristics are integrated backward from the grid points. This implies that in the first step (1) the grid point $G$ has to coincide with the intermediate point $G=\left(D_{x}^{(1)}, S_{y}^{(1)}\right)$, or equivalently $S^{(1)}=\left(S_{x}^{(1)}, G_{y}\right)$ and $D^{(1)}=\left(G_{x}, D_{y}^{(1)}\right)$. We have displayed these characteristics in Figure 3. In this way the distribution function has been shifted along $v_{x}$ according to

$$
f^{\text {inter }}\left(G_{x}, G_{y}\right)=f^{\text {old }}\left(S_{x}^{(1)}, G_{y}\right)
$$

Given a sufficiently smooth behaviour of the characteristics we can assume that the interpolation scheme causes all other points of the distribution function 


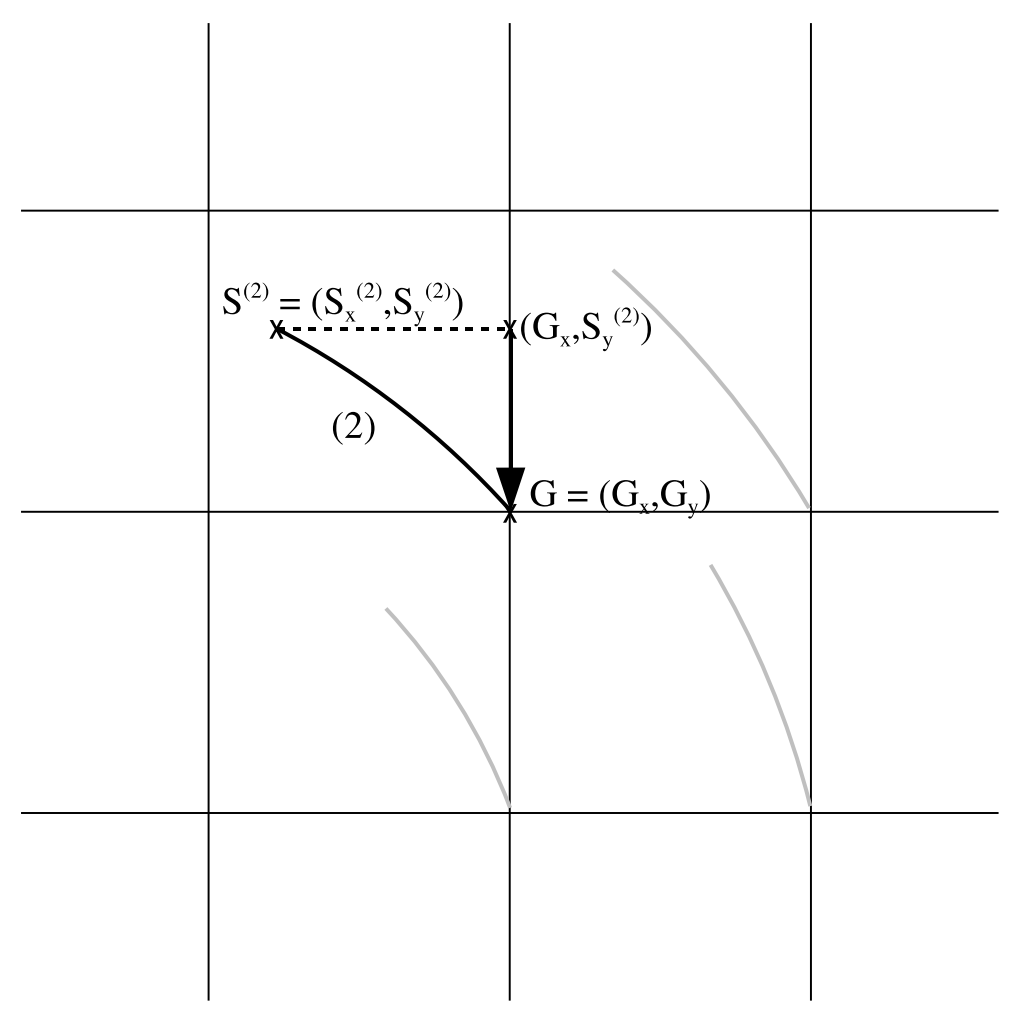

Fig. 4. Schematic diagram of the second step of integration of the characteristics using the backsubstitution algorithm. The distribution function is shifted in the $v_{y}$-direction from the intermediate point to the destination point $G$ of the characteristic. Gray lines show corresponding characteristics in the other cells.

to be shifted accordingly. This is particularly true for the characteristic that ends in the grid point $G$ (dashed line in Figure 3). This characteristic will be important in the following step.

In the second step (Figure 4) we, therefore, need to choose the characteristic that ends in $G$. Then the source point $S^{(2)}$ is given by $S^{(2)}=X(t-\Delta t, t, G)$. The shift is performed in the $v_{y}$ direction so that

$$
f^{\text {new }}\left(G_{x}, G_{y}\right)=f^{\text {inter }}\left(G_{x}, S_{y}^{(2)}\right) .
$$

Since in the first step we had (assuming again correct interpolation)

$$
f^{\text {inter }}\left(G_{x}, S_{y}^{(2)}\right)=f^{\text {old }}\left(S_{x}^{(2)}, S_{y}^{(2)}\right)
$$

we finally have

$$
f^{\text {new }}\left(G_{x}, G_{y}\right)=f^{\text {old }}\left(S_{x}^{(2)}, S_{y}^{(2)}\right)
$$

We now use this motivation to write down a general scheme for threedimensional velocity space. For every grid point $G$ we perform the integration in three one-dimensional substeps, one for each component $v_{x}, v_{y}, v_{z}$. For each integration a source coordinate $S_{x}^{(1)}, S_{y}^{(2)}$ and $S_{z}^{(3)}$ is calculated from a 
characteristic which does not necessarily pass through $G$. To find $S_{x}^{(1)}$ for the $v_{x}$-integration we demand

$$
\begin{aligned}
G_{x} & =D_{x}^{(1)}, \\
G_{y} & =\left(X\left(t-\Delta t, t, D^{(1)}\right)\right)_{y}, \\
G_{z} & =\left(X\left(t-\Delta t, t, D^{(1)}\right)\right)_{z} .
\end{aligned}
$$

In general this is a nonlinear system of equations for the components $D_{y}^{(1)}$ and $D_{z}^{(1)}$. The details of this system depend on the way the characteristics are calculated. Given $D^{(1)}$ one then has

$$
S_{x}^{(1)}=\left(X\left(t-\Delta t, t, D^{(1)}\right)\right)_{x},
$$

and the integration can be performed from $S_{x}^{1}$ to $G_{x}$ in the $v_{x}$-direction.

Similarly we demand for the $v_{y}$-integration

$$
\begin{aligned}
G_{x} & =D_{x}^{(2)}, \\
G_{y} & =D_{y}^{(2)} \\
G_{z} & =\left(X\left(t-\Delta t, t, D^{(2)}\right)\right)_{z} .
\end{aligned}
$$

$D^{(2)}$ differs from $G$ only in the $v_{z}$-component. Once $D_{z}^{(2)}$ is found we have

$$
S_{y}^{(2)}=\left(X\left(t-\Delta t, t, D^{(2)}\right)\right)_{y} .
$$

Again the integration is now performed from $S_{y}^{(2)}$ to $G_{y}$ in the $v_{y}$-direction.

The $v_{z}$-integration finally is straightforward. Since $D^{(3)}=G$ we have

$$
S_{z}^{(3)}=(X(t-\Delta t, t, G))_{y}
$$

and the integration is performed from $S_{z}^{(3)}$ to $G_{z}$ in the $v_{z}$-direction.

We want to emphasise that this scheme integrates the characteristics exactly, which means that in terms of Figs. 1 and 2 the backsubstitution scheme has errors which are exactly zero.

\section{Application to Boris scheme}

The main task now is to calculate the characteristics or their approximations in the presence of a magnetic field. A commonly used approach is the Boris 
scheme [9]. Here the integration step is formulated as an implicit finite difference scheme

$$
\frac{\mathbf{v}^{n+1}-\mathbf{v}^{n}}{\Delta t}=\frac{q}{m}\left(\mathbf{E}+\frac{\mathbf{v}^{n+1}+\mathbf{v}^{n}}{2} \times \mathbf{B}\right)
$$

The electric and magnetic forces are separated,

$$
\begin{aligned}
& \mathbf{v}^{-}=\mathbf{v}^{n}+\frac{\Delta t}{2} \frac{q}{m} \mathbf{E}, \\
& \mathbf{v}^{+}=\mathbf{v}^{n+1}-\frac{\Delta t}{2} \frac{q}{m} \mathbf{E},
\end{aligned}
$$

leading to

$$
\frac{\mathbf{v}^{+}-\mathbf{v}^{-}}{\Delta t}=\frac{q}{2 m}\left(\mathbf{v}^{+}+\mathbf{v}^{-}\right) \times \mathbf{B}
$$

The transformation from $\mathbf{v}^{-}$to $\mathbf{v}^{+}$is a pure rotation with an angle $\theta$ where

$$
\left|\tan \frac{\theta}{2}\right|=\frac{\Delta t}{2} \frac{q B}{m} .
$$

For small angles $\theta$ this is close to the exact angle $\theta_{\text {exact }}=\Delta t q B / m$.

In contrast to the original scheme of Boris, we aim to trace the characteristics backward in time. This means we want to find $\mathbf{v}^{-}$in terms of $\mathbf{v}^{+}$. We thus reverse the original scheme and rotate $\mathbf{v}^{+}$by $\vartheta$. To implement this rotation the vectors $\mathbf{t}$ and $\mathbf{s}$ are defined

$$
\mathbf{t}=\frac{-\Delta t}{2} \frac{q \mathbf{B}}{m}, \quad \mathbf{s}=\frac{2 \mathbf{t}}{1+t^{2}}
$$

Then the rotation is performed in two steps

$$
\mathbf{v}^{\prime}=\mathbf{v}^{+}+\mathbf{v}^{+} \times \mathbf{t}
$$

and

$$
\mathbf{v}^{-}=\mathbf{v}^{+}+\mathbf{v}^{\prime} \times \mathbf{s}
$$

This scheme now supplies $S=\mathbf{v}^{n}=\left(v_{x}^{n}, v_{y}^{n}, v_{z}^{n}\right)$ in terms of $D=\mathbf{v}^{n+1}=$ $\left(v_{x}^{n+1}, v_{y}^{n+1}, v_{z}^{n+1}\right)$. To facilitate the further calculations we insert (37) into (38) and separate $\mathbf{v}^{-}$into it's components

$$
\begin{aligned}
& v_{x}^{-}=\left(1-s_{y} t_{y}-s_{z} t_{z}\right) v_{x}^{+}+\left(s_{y} t_{x}+s_{z}\right) v_{y}^{+}+\left(s_{z} t_{x}-s_{y}\right) v_{z}^{+}, \\
& v_{y}^{-}=\left(s_{x} t_{y}-s_{z}\right) v_{x}^{+}+\left(1-s_{x} t_{x}-s_{z} t_{z}\right) v_{y}^{+}+\left(s_{z} t_{y}+s_{x}\right) v_{z}^{+}, \\
& v_{z}^{-}=\left(s_{x} t_{z}+s_{y}\right) v_{x}^{+}+\left(s_{y} t_{z}-s_{x}\right) v_{y}^{+}+\left(1-s_{x} t_{x}-s_{y} t_{y}\right) v_{z}^{+}
\end{aligned} .
$$

We now need to solve the systems of equations $(22-24)$ and $(26-28)$ for the first and the second backsubstitution step. As stated before, the third step 
is straightforward since $D^{(3)}$ is already known. The complete problem can be written in the form

$$
\begin{aligned}
v_{x}^{n} & =v_{x}^{n}\left(v_{x}^{n+1}, v_{y}^{n}, v_{z}^{n}\right), \\
v_{y}^{n} & =v_{y}^{n}\left(v_{x}^{n+1}, v_{y}^{n+1}, v_{z}^{n}\right), \\
v_{z}^{n} & =v_{z}^{n}\left(v_{x}^{n+1}, v_{y}^{n+1}, v_{z}^{n+1}\right) .
\end{aligned}
$$

Since the bijections between $v^{n}$ and $v^{-}$, on one hand, and $v^{n+1}$ and $v^{+}$, on the other hand, are trivial (see eqs (32) and (33)) it is sufficient to formulate the three steps

$$
\begin{aligned}
& v_{x}^{-}=v_{x}^{-}\left(v_{x}^{+}, v_{y}^{-}, v_{z}^{-}\right), \\
& v_{y}^{-}=v_{y}^{-}\left(v_{x}^{+}, v_{y}^{+}, v_{z}^{-}\right), \\
& v_{z}^{-}=v_{z}^{-}\left(v_{x}^{+}, v_{y}^{+}, v_{z}^{+}\right) .
\end{aligned}
$$

To find (45) we take eqs (40) and (41) and solve for $v_{y}^{+}$and $v_{z}^{+}$giving

$$
\begin{aligned}
& v_{y}^{+}=\frac{\left(1-s_{x} t_{x}-s_{y} t_{y}\right) v_{y}^{-}-\left(s_{x}+s_{z} t_{y}\right) v_{z}^{-}+\left(s_{x} t_{y}(m-1)-s_{x} n_{y}+s_{x} s_{y}+s_{z}\right) v_{x}^{+}}{m\left(s_{x} t_{x}-1\right)+1+s_{x}\left(s_{x}-t_{x}-n_{x}\right)} \\
& v_{z}^{+}=\frac{\left(s_{x}-s_{y} t_{z}\right) v_{y}^{-}+\left(1-s_{x} t_{x}-s_{z} t_{z}\right) v_{z}^{-}+\left(s_{x} t_{z}(m-1)-s_{x} n_{z}-s_{y}+s_{x} s_{z}\right) v_{x}^{+}}{m\left(s_{x} t_{x}-1\right)+1+s_{x}\left(s_{x}-t_{x}-n_{x}\right)}
\end{aligned}
$$

where $m=\mathbf{s} \cdot \mathbf{t}$ and $\mathbf{n}=\mathbf{s} \times \mathbf{t}$. These can be inserted into (39) which then provides the expression (45) for the first step.

For the second step (46) only eq (41) has to be solved for $v_{z}^{+}$giving

$$
v_{z}^{+}=\frac{-\left(s_{y}+s_{x} t_{z}\right) v_{x}^{+}+\left(s_{x}-s_{y} t_{z}\right) v_{y}^{+}+v_{z}^{-}}{1-s_{x} t_{x}-s_{y} t_{y}} .
$$

With this, $v_{z}^{+}$can be substituted in eq (40) giving $v_{y}^{-}$in the form (46).

By virtue of equation (41) the $z$-component is already given in the form (47). Thus, no further calculation has to be done for the third step.

Finally we want to discuss the error of Boris' scheme combined with the backsubstitution method. We again investigate the same problem as formulated in section 3 where the velocity vector is rotated around the origin. While Boris' scheme introduces a phase error in this rotation, the magnitude of the velocity is preserved. Using this combined scheme in a grid based Vlasov solver implies that the only diffusion in the system originates from the reconstruction of the distribution function. 


\section{Bernstein Waves}

We have applied the schemes described above to the simulation of Bernstein waves in a periodic system. These are electrostatic waves propagating at a right angle to a given constant magnetic field $[10,11]$. The ions are treated as a static neutralising background, while the electrons oscillate in the electrostatic field. We assume that $\mathbf{B}=B_{z} \hat{\mathbf{z}}$ and the wavevector $\mathbf{k}=k_{x} \hat{\mathbf{x}}$. Then the dispersion relation can be written as

$$
1+\frac{2 \omega_{p e}^{2}}{\sqrt{\pi} \Omega^{2}} \sum_{n=0}^{\infty} A_{n}(w) k^{2 n} \frac{M\left(n+3 / 2,2 n+2,-k^{2}\right) \Gamma(n+1.5)}{\Gamma(2 n+2)}=0
$$

with

$$
A_{n}(w)=\frac{(2 n+1)\left(n^{2}+n-w^{2}\right)}{\left(n^{2}-w^{2}\right)\left((n+1)^{2}-w^{2}\right)} .
$$

Here we used $w=\omega / \Omega, k=v_{t h} k_{x} / \Omega, \Omega=e B_{z} / m_{e}$ is the electron cyclotron frequency and $\omega_{p e}$ is the electron plasma frequency. $m_{e}$ is the electron mass and $e$ is the electron charge. $\Gamma$ is the gamma function and $M$ is Kummer's confluent hypergeometric function. We chose $\omega_{p e}^{2}=\Omega^{2}$ for all simulations. For a given $k_{x}$, the above dispersion relation has an infinite number of solutions for $\omega$. We performed the simulations in one space and three velocity dimensions, $\left(x, v_{x}, v_{y}, v_{z}\right)$. Although two velocity dimensions would be sufficient for this system, we keep the $v_{z}$-dimension to make the results transferable to electromagnetic simulations in which the magnetic field is not fixed. The simulation box has a length $L$ which was resolved with 64 grid cells. The velocity space was sampled with 50 grid cells in each direction in the interval from $-4 v_{t h}$ to $+4 v_{t h}$. The length of the box is chosen to $L=2 \pi / k_{x}$ so that exactly one wavelength of the Bernstein mode fits into the system. In this way the size of a grid cell in space is $\Delta x=2 \pi / 64 k_{x}$. The timestep was chosen such that the CFL-condition is satisfied $\Delta t=\Delta x / 5 v_{t h}$. For the integration of the distribution function on the grid we use a flux conservative and positive scheme $[7]$.

The first simulation was initialised with the Bernstein wave of the lowest frequency mode $1 \leq \omega / \Omega<2$. Runs were performed for different values of $k_{x}$ and using the different integration schemes. The frequency of the wave was then determined using a Fourier analysis. The results are shown in Fig. 5 for the time-splitting scheme A, time-splitting scheme B, backsubstitution using the Boris scheme and backsubstitution using the exact characteristics. We can observe that the time-splitting scheme A clearly shows the largest error in the dispersion of the waves. The errors of all the other schemes appear comparable and are very good for all values of $k \geq 0.15$. The larger errors for

smaller wavenumbers are due to the choice of the timestep $\Delta t$. Inserting the 


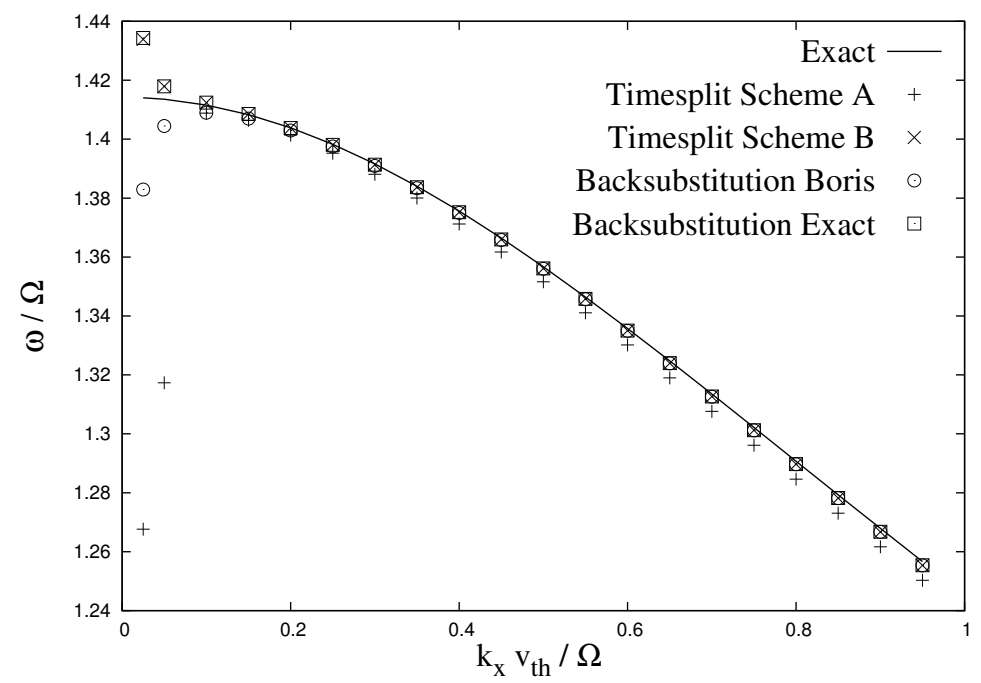

Fig. 5. Comparison of the dispersion of Bernstein waves between the exact result and the Vlasov simulation using different schemes for integrating the characteristics.

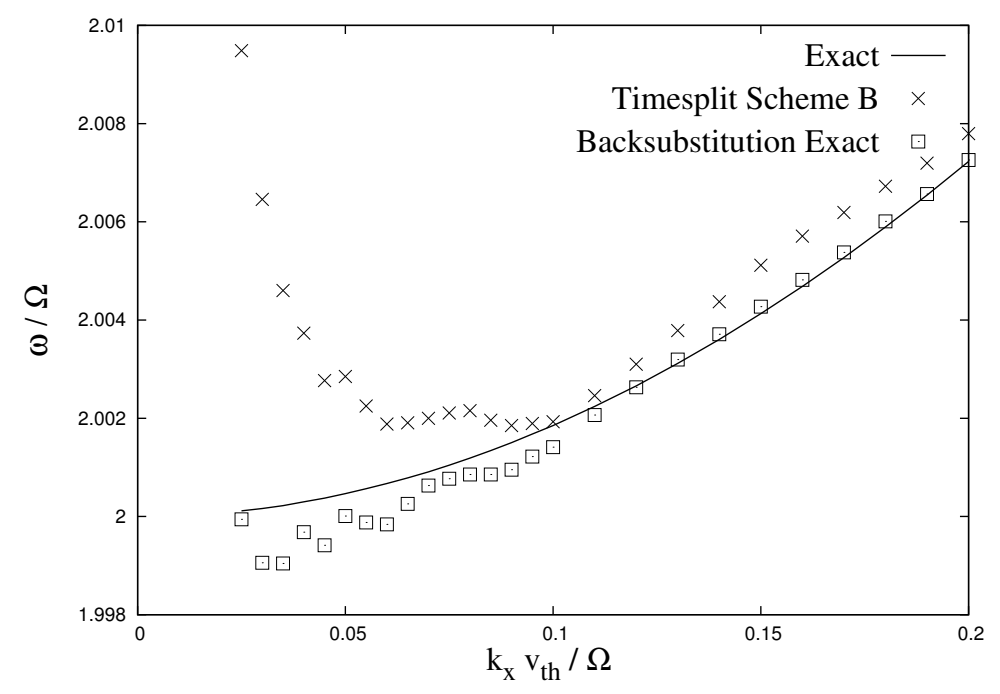

Fig. 6. Comparison of the dispersion of Bernstein waves between the exact result and the Vlasov simulation using different schemes for integrating the characteristics.

definitions for $\Delta x$ and $k$ one finds that

$$
\Delta t=2 \pi \Omega^{-1} \frac{1}{320 k} .
$$

For $k=0.05$ this means $\Delta t=2 \pi \Omega^{-1} / 16$ or 16 steps for one gyration.

In another simulation run, the second Bernstein mode $2 \leq \omega / \Omega<3$ was initialised. Fig. 6 shows the result of the time-splitting scheme $B$ and the backsubstitution method with exact integration of the characteristics. The dispersion relation is shown for values of $k \leq 0.2$, where the errors are largest 
Table 1

\begin{tabular}{|c|c|}
\hline Scheme & Comp. time / min \\
\hline \hline Splitting Scheme A & 192 \\
\hline Splitting Scheme B & 165 \\
\hline Backsubs. Boris & 94 \\
\hline Backsubs. Exact & 137 \\
\hline
\end{tabular}

Computational time used for a typical run

due to the choice of the timestep. For this case we observe that the backsubstitution show superior results when compared to the time-splitting method.

Finally, we want to look at the computational time used by the different schemes. Table 1 shows the times used for a typical run. The runs for the different schemes were carried out with exactly the same conditions on the same machine. Here we find a clear advantage of the backsubstitution scheme over the splitting schemes. The backsubstitution scheme with Boris integration of the characteristics reduces the computational effort by more than $50 \%$ when compared to the time splitting scheme A. With time-splitting scheme B this improvement is still approximately $43 \%$. The timing for the exact backsubstitution shows less improvement due to the fact that trigonometric functions have to be evaluated. The reason for the speed-up is the fact that the backsubstitution method has to integrate the distribution function only once for each velocity dimension $v_{x}, v_{y}$ and $v_{z}$. The splitting schemes, on the other hand, have to integrate the distribution function 7 times. Although the numerical effort of integrating the characteristics in each step is considerably smaller in the splitting scheme B, this is only a part of the computational time spent. Other parts involve the interpolation of the distribution function and the calculation of fluxes across the cell boundaries. Considering all the above results, the backsubstitution method together with the Boris scheme can be taken as a good alternative to the traditional time splitting method if speed is the major issue. To obtain the most accurate results, the backsubstitution method together with the exact integration of the characteristics is the superior scheme. In addition it also is slightly faster than the time-splitting scheme.

\section{Conclusions}

We have shown that the time splitting method for integrating Vlasov's equation in higher dimensions can introduce systematic errors when used in the presence of a magnetic field. These errors originate from the effective splitting of the integration of the characteristics, when a higher order integration scheme is used. The errors cause the temperature of the distribution function 
to increase over time, and thus artificially feed energy into the system.

The backsubstitution method presented here for the general case of arbitrary integration schemes of the characteristics eliminates this problem. Here not those characteristics that pass through the grid point are integrated, but those characteristics that will give a consistent scheme when executed in sequence for the full timestep. This not only provides the best accuracy possible but also reduces the number of integration steps. While in three dimensional velocity space the time-splitting scheme consists of 7 steps, the backsubstitution method only uses 3 steps since each component needs to be integrated only once. Due to this advantage the backsubstitution method together with the Boris scheme typically decreases the computational effort by over $40 \%$ as compared to a simple time splitting method while the errors remain small. On the other hand, highest accuracy can be achieved with the backsubstitution method together with the exact integration of the characteristics.

\section{Acknowledgements}

Access to the JUMP multiprocessor computer at the FZ Jülich was made available through project HBO20. This work was supported by the SFB 591 of the Deutsche Forschungsgesellschaft.

\section{References}

[1] C. Z. Cheng, G. Knorr, The integration of the Vlasov equation in configuration space, J. Comp. Phys. 22 (1976) 330.

[2] A. Arakawa, Computational Design for Long-Term Numerical Integration of the Equations of Fluid Motion: Two dimensional Incompressible Flow, Part 1, J. Comp. Phys. 1 (1966) 119. Reprinted in J. Comp. Phys. 135 (1997) 103

[3] E. Fijalkow, A numerical solution to the Vlasov equation, Comput. Phys. Communications 116 (1999) 319.

[4] E. Sonnendrücker, J. Roche, P. Bertrand, a. Ghizzo, The Semi-Lagrangian Method for the Numerical Resolution of Vlasov Equations, J. Comp. Phys. 149 (1998) 201

[5] F. Filbet, E. Sonnendrücker, Comparison of Eulerian Vlasov Solvers, Comput. Phys. Communications 150 (2003) 247.

[6] F. Califano, A. Mangeney, C. Cavazzoni, P. Travnicek, A numerical scheme for the integration of the Vlasov-Maxwell system of equations, in: Science and Supercomputing at CINECA, 2001, p. 456. 
[7] F. Filbet, E. Sonnendrücker, P. Bertrand, Conservative numerical schemes for the Vlasov equation, J. Comp. Phys. 172 (2001) 166.

[8] T. Arber, R. G. L. Vann, A critical comparison of Eulerian grid based Vlasov solvers, J. Comp. Phys. 180 (2002) 339.

[9] C. K. Birdsall, A. B. Langdon, Plasma Physics via Computer Simulation, McGraw-Hill, New York, 1985.

[10] D. R. Nicholson, Introduction to plasma theory, John Wiley \& Sons, New York, 1983.

[11] I. B. Bernstein, Waves in a Plasma in a Magnetic Field, Phys. Rev. 109 (1958) 10 . 